\title{
Design of Air Conditioning Remote Controller Based on Spontaneous EEG
}

\author{
Jinghai Yin and Yuhua Chen
}

Institute of Information Technology, Jiangxi University of Technology, Nanchang 330098, China

\section{Keywords: EEG; Air conditioning; Remote controller}

\begin{abstract}
This article describes the design of a device which can according to different users brain wave characteristics to choose the most suitable for the user to air temperature, but also can automatically adjust the wind speed reached users the most suitable to accept the degree of, all regulatory processes are by the device automatic implementation and completion of, the air conditioning of the refrigeration or heating process of brain waves to achieve adaptive.
\end{abstract}

\section{Introduction}

EEG based brain computer interface (BCI) systems can be used for people with disabilities to improve their quality of life. Applications of BCI systems comprise the restoration of movements, communication and environmental control. The most interesting thing in these applications is to control the roles in game by player's EEG. A volume of research has been performed on create information chain from brain to PC game by BCI system. Anton Nijholt(2008) Reported that Prototype BCI applications now appear in the domain of games and entertainment that aim at adapting and controlling a game using brain signals in addition to traditional physical and mental abilities.

In daily life, the air conditioning is we often want to use household appliances, from everyone's bedroom to the various units of the office, or the large and small all types of hotels, air conditioning has been very widely used, their most basic control functions are basically the same, including control of the temperature and the selection of wind speed. Due to the body during wakefulness required temperature and sleep when the proper temperature usually have a certain gap, so it is easy to cause people in sleep is to blow the air conditioning cold, and rely on spontaneous EEG changes of adjusting these parameters of the equipment will be able to solve the corresponding problem.

\section{Methods}

In this paper, we use short-term Fourier transform for the adaptive time-frequency analysis and energy entropy have been applied to feature extraction.

Shannon entropy. The conventional definition of the Shannon entropy was described in terms of the temporal distribution of signal energy in a given time window. The distribution of energy in a specified number of data values intervals was described in terms of the probabilities in signal space $\left\{p_{i}\right\}$ where $p_{i}$ was the probability that $X=a_{i}$, so entropy for discrete random variable $X$ was defined as

$$
H(X)=-\sum_{i} p_{i} \log \left(p_{i}\right)
$$

The entropy of a random variable reflected the degree of disorder that the variable possessed. The more uncertain the variable was, the greater its entropy.

Energy entropy. Short-term Fourier transform was a time-frequency analysis, which could analyze no stationary time-varying signals on frequency domain and time domain at the same time, so the 
dynamic change of signal energy with time could be observed. Energy Entropy defined on the basis of this could characterize signal complexity with the changes in time, and also many of the characteristics in frequency domain, which had a good time-frequency local capabilities.

Let $E_{1}, E_{2}, \wedge E_{m}$ represented energy distribution on the $m$ frequency bands. Then signal energy $E$

equaled to the sum of $E_{j}$ in a time window $E=\sum_{j=1}^{m} E_{j}$, where $E_{j}=\sum_{k}\left|D_{j}(k)\right|^{2}$, where $D_{j}$ was spectrum on the jth frequency band. Let

$$
\begin{aligned}
& p_{j}=E_{j} / E \\
& W_{e}=-\sum_{j} p_{j} \log p_{j}
\end{aligned}
$$

\section{System Data Flow and workflow}

The data flow diagram of the system is as follows:

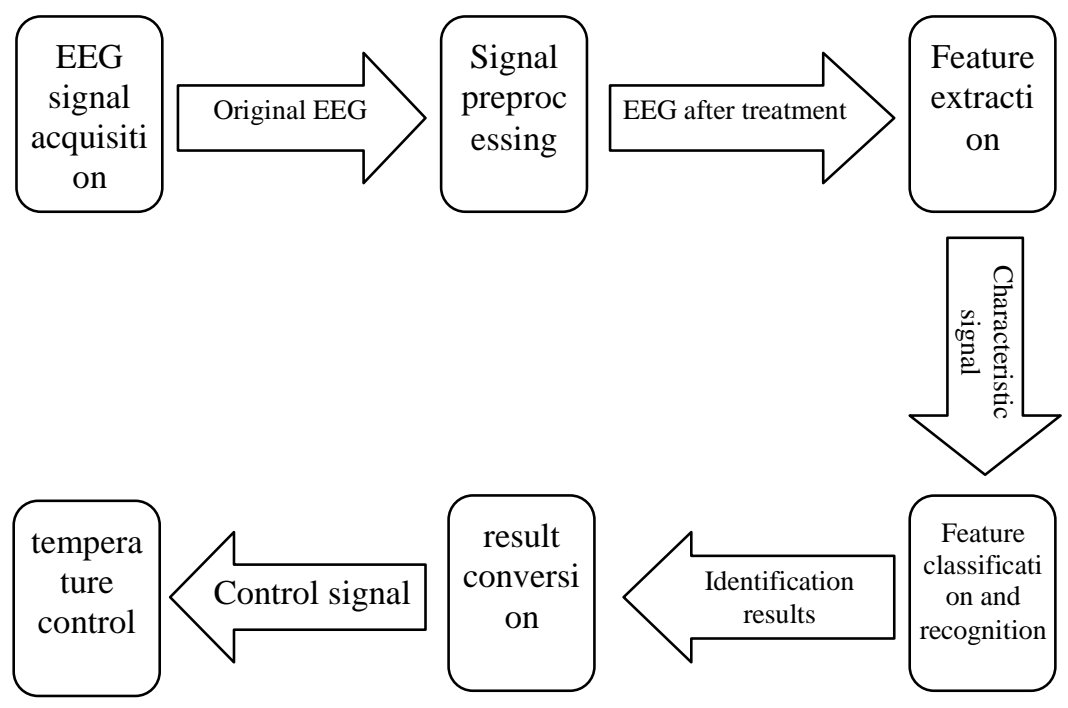

Fig. 1 Data flow chart

As Fig. 1 shows, First of all, through the acquisition of brain electrical signal acquisition of the original brain electrical signal, and then through the signal preprocessing to the brain electrical signal processing, including filtering and denoising, etc. Then the preprocessed signal for feature extraction, and produce the characteristic signal classification and recognition, the final recognition result after the conversion obtained control signal via the remote control transmitter to control the air conditioning running. 


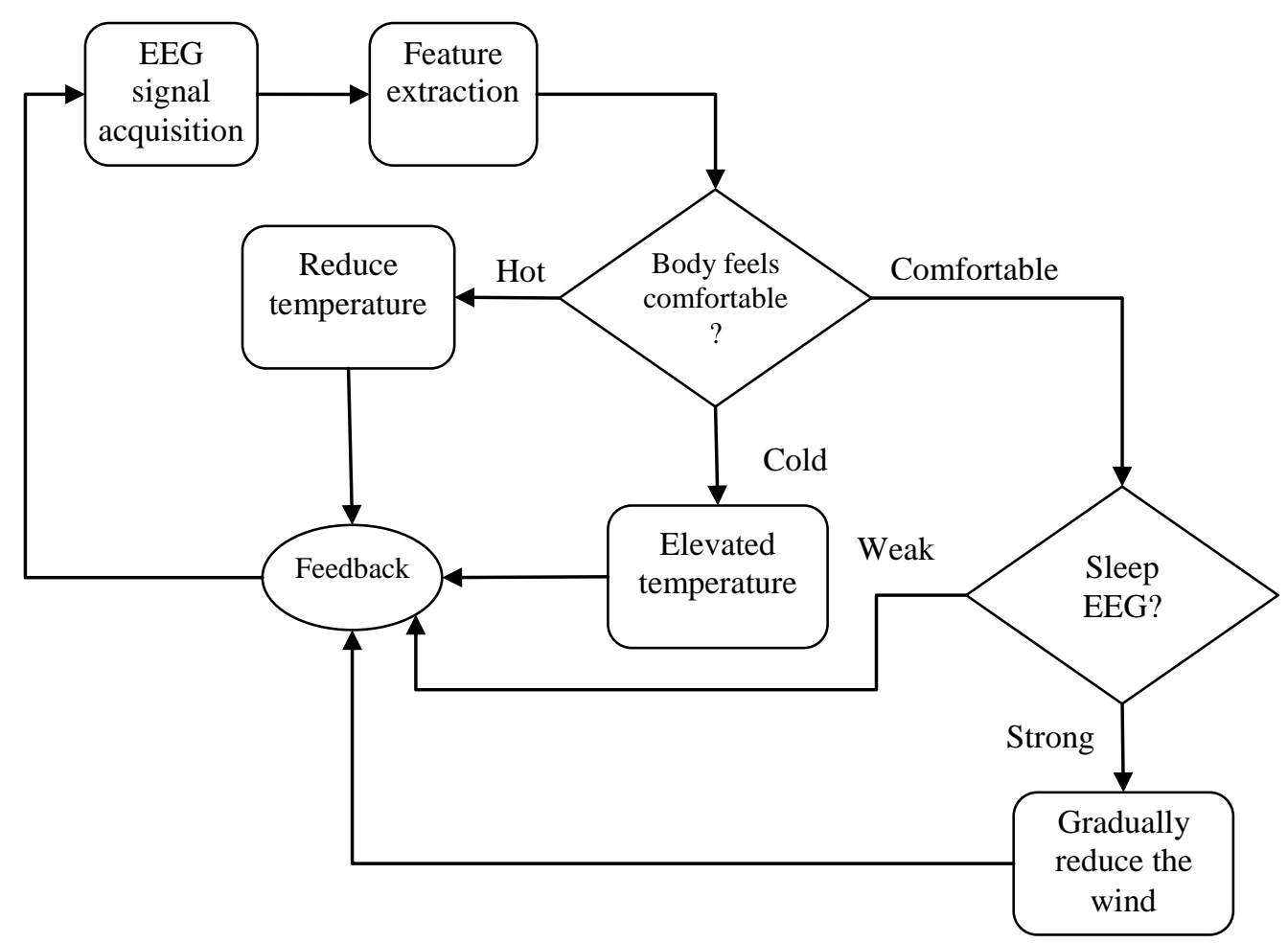

Fig. 2 workfolw chart

As shown in Figure two, when we open the air conditioning control system, wear a brain power acquisition device. System through the identification of brain waves to determine the brains of the current user comfort to select the appropriate temperature, when the brain feel hot automatically, reduce air conditioning temperature, when the brain to feel the cold is automatically increased air conditioning temperature, when feel more comfortable, system will be in accordance with the current set of temperature continues to run. When the system determines the current user of the brain in the comfortable temperature state, the system detecting brain waves contain sleeping wave component. When the system is found relatively strong sleep wave signal, indicating that users have begun to enter the sleep state, the system will gradually reduce the wind force and make the corresponding adjustment to the temperature at, to ensure that the user does not because of blowing air and cold.

\section{Design of System architecture}

The external structure of the system is shown in the following figure: 


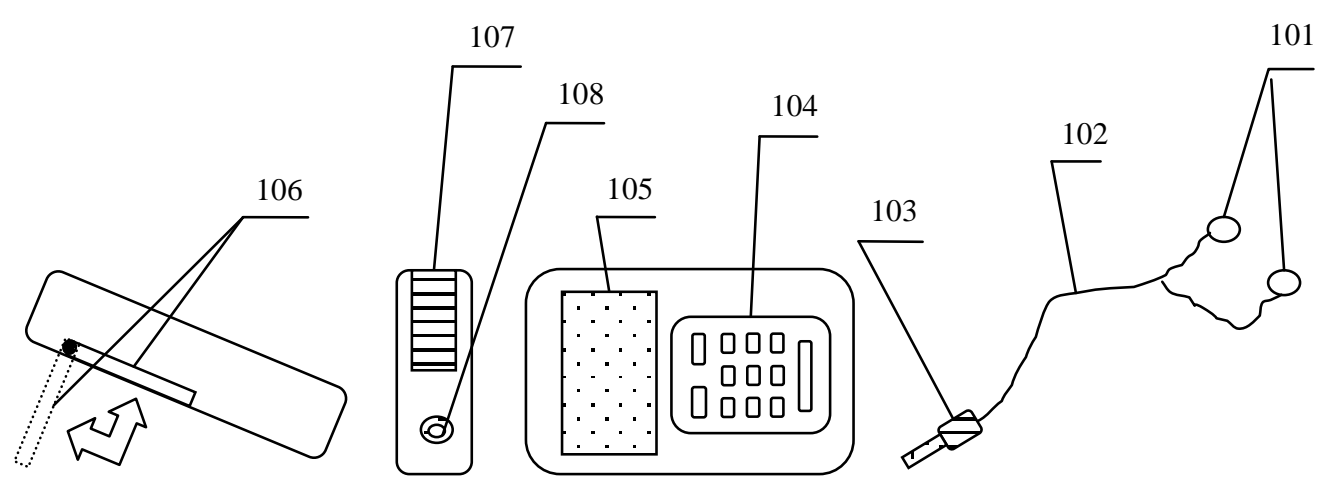

Fig. 3 External structure diagram

As shown in Fig. 3, EEG adaptive air conditioning remote control device is composed of two parts, including the remote control part and the EEG signal acquisition part, between the two parts of the interface signals realizes transmission and receive information.

Brain electrical signal acquisition and analysis is made up of two dry electrodes 102, signal wires 101 and a brain electrical signal plug 103. Dry electrode 101 is an alloy material, which is attached to the surface of the skin. Two electrodes placed on the user's left prefrontal cortex and right prefrontal area, the system through the two dry electrodes to collect the original EEG signals, and then through the two signal conductors 102 will the conduction to the EEG signal plug 103 to output EEG signal.

Remote control is composed of remote control panel 104, liquid crystal display 105 , retractable bracket 106, a battery cover 107, and EEG signal interface 108. Remote control panel 104 is located in the front of the remote controller, contains a series of control air conditioning operation keys. These keys compared with conventional air-conditioning controller is not big difference, the only difference is increased by a brain electrical control switch button, the remote control to the front of the upper part of the liquid crystal display 105 for indicating the working state of the remote control. Retractable bracket 106 for supporting the remote controller at the remote control air conditioning, at the bottom of the battery cover 107 and EEG interface 108.

\section{Discussion}

The system can accord the change of brain waves to determine the current user for the optimum temperature, when users are busy with their work; the system can accord the temperature of its users to feel the temperature of the automatic adjustment of the air conditioner. Also for open air conditioning to sleep of the user, the system will according to the real-time sleep EEG changes gradually the temperature correction and to reduce the wind.

\section{Acknowledgment}

This work was supported by Jiangxi province department of science and technology support project [20142bbe50030] and Natural Sciences Project of Jiangxi Science and Technology Department [20122BAB201049]. The authors are grateful for the anonymous reviewers who made constructive comments. 


\section{References}

[1] G. Pfurtscheller, C. Guger, G. Müller, G. Krausz, and C. Neuper, Brain oscillations control hand orthosis in a tetraplegic,“"Neurosci. Lett., vol. 292, pp. 211-214, 2000.

[2] S.M. Stevens and S. Saldamarco (Eds.) BCI for Games: A 'State of the Art' Survey ICEC 2008, LNCS 5309, pp. 225-228, 2008.

[3] Ince N.F., Arica S., Tewfik A., "Classification of single trial motor imagery EEG recordings with subject adapted nondyadi arbitrary time-frequency tilings," J Neural Eng, Vol. 3, pp. 235-244, 2006.

[4] Jianfeng Hu,Dan Xiao,Zhendong Mu. Application of Energy Entropy in Motor Imagery EEG Classification. International Journal of Digital Content Technology and its Applications,2009,3(2): 83-90

[5] R. Nicole, "Title of paper with only first word capitalized," J. Name Stand. Abbrev., in press.

[6] McFarland D.J., Wolpaw J.R., "Sensorimotor rhythm-based brain-computer interface (BCI): model order selection for autoregressive spectral analysis," J Neural Eng, Vol. 5, pp. 155-162, 2008.

[7] Ramoser H., Müller-Gerking J., Pfurtscheller G., "Optimal spatial filtering of single trial EEG during imagined hand movement," IEEE Trans Rehabil Eng, Vol. 8, pp. 441-446, 2000.

[8] Müller-Gerking J., Pfurtscheller G., Flyvbjerg H., "Designing optimal spatial filters for single-trial EEG classification in a movement task," Clin Neurophysiol, Vol. 110, pp. 787-798, 1999.

[9] Hoffmann U., Vesin J.M., Ebrahimi T., "Spatial filters for the classification of event-related potentials," Proc 14th Eur Symp Artif Neural Networks, pp. 47-52, 2006.

[10]Liao X., Yao D.Z., Wu D. et al, "Combining Spatial Filters for the Classification of Single-Trial EEG in a Finger Movement Task,” IEEE Trans Biomed Eng, Vol. 54, pp. 821-831, 2007.

[11]Hinterberger T., Kubler A., Kaiser J., "A brain-computer interface (BCI) for the locked-in: comparison of different EEG classifications for the thought translation device," Clin Neurophys, Vol. 114 , pp. 416-425, 2003.. 